\title{
Common fixed point theorems for multimaps in locally convex spaces and some applications
}

\author{
HR Goudarzi', Hassan Azadi Kenary ${ }^{1}$ and Choonkil Park ${ }^{2 *}$
}

\section{"Correspondence:}

baak@hanyang.ac.kr

2Department of Mathematics,

Research Institute for Natural

Sciences, Hanyang University, Seoul,

133-791, South Korea

Full list of author information is

available at the end of the article

\begin{abstract}
In this paper some fixed point theorems for multimaps on locally convex spaces have been introduced. As their applications, some well-known fixed point theorems will be deduced. Also, we see a notable result in differential equations.
\end{abstract}

AMS Subject Classification: 46A55; 46B99

Keywords: fixed points; locally convex spaces; integral equations; multimap

\section{Introduction}

Fixed point theorems have been studied by many authors. Probably the oldest fixed point theorem is the contraction mapping principle. From this theorem, the study of fixed point theorems arose in different settings. More than a thousand of fixed point theorems have been studied and proved by many authors. Among them, there are some notable cases such as Brower's, Schauder-Tychonov's, Markov-Kakutani's and many others. Although many fixed point theorems have been flourishing for both single maps and groups of maps in metric and Banach spaces, only a few have been reported in general topological vector spaces (for example, see [1,2] and [3]). Recently, set-valued maps (or multimaps) have been very important tools in non-linear analysis; e.g., see [4] and [5]. Some existence results have been generalized and extended to multimaps in general spaces (as an example, see [6]).

In this paper, some new generalized fixed point theorems for multimaps in locally convex spaces have been proved, and as their results, we imply some notable theorems such as Markov-Kakutani and Schauder fixed point theorems. Finally, we see an application of the proved results in differential equations.

\section{Preliminaries}

Let $(X, \tau)$ be a Hausdorff locally convex space. A family $\left\{p_{\alpha}: \alpha \in I\right\}$ of seminorms on $X$ is called an associated family for $\tau$ if the family $\{\gamma U: \gamma>0\}$ forms a base of neighborhoods of zero for $\tau$, where $U=\bigcap_{i=1}^{n} U_{\alpha_{i}}$ and $U_{\alpha_{i}}=\left\{x: p_{\alpha_{i}}(x)<1\right\}$. A family $\left\{p_{\alpha}: \alpha \in I\right\}$ of seminorms on $X$ is called an augmented associated family for $\tau$ if $\left\{p_{\alpha}: \alpha \in I\right\}$ is an associated family such that $\max \left\{p_{\alpha}, p_{\beta}\right\} \in\left\{p_{\alpha}: \alpha \in I\right\}$ for any $\alpha, \beta \in I$. We will denote by $\mathcal{A}(\tau)$ and $\mathcal{A}^{*}(\tau)$ the associated and augmented associated seminorms $\left\{p_{\alpha}: \alpha \in I\right\}$, respectively. As a wellknown result, there always exists a family $\left\{p_{\alpha}: \alpha \in I\right\}$ of seminorms on $X$ such that $\left\{p_{\alpha}\right.$ : 
$\alpha \in I\}=\mathcal{A}^{*}(\tau)$ (see [7,p.203]). A subset $M$ of $X$ is $\tau$-bounded in $X$ if and only if each $p_{\alpha}$ is bounded on $M$.

A subset $A \subseteq X$ is said to be star-shaped if there exists an element $x \in A$ such that $t x+$ $(1-t) y \in A$ for all $t \in[0,1]$ and for all $y \in A$. Such an element is called a star-point of $A$. The set of all star-points of $A$ is called the star-core of $A$.

Let $C$ be a nonempty convex subset of $X$ and $q \in C$. A self-mapping $T$ on $C$ is said to be affine if

$$
T(\lambda x+(1-\lambda) y)=\lambda T(x)+(1-\lambda) T(y)
$$

for all $x, y \in C$ and $\lambda \in(0,1)$. Also, $T$ is called to be affine with respect to $q$ if

$$
T(\lambda x+(1-\lambda) q)=\lambda T(x)+(1-\lambda) T(q)
$$

for all $x \in C$ and $\lambda \in(0,1)$. There is an example of an affine mapping with respect to a point which is not affine (see [8]).

Let $A$ be a subset of $X$. We will denote by $2^{A}$ the family of all subsets of $A$. Let $X, Y$ be topological vector spaces and let $T: X \longrightarrow 2^{Y}$ be a multimap. $T$ is said to be convex [9] if for each $x_{1}, x_{2} \in X$ and each $\lambda \in[0,1]$, we have

$$
\lambda T\left(x_{1}\right)+(1-\lambda) T\left(x_{2}\right) \subseteq T\left(\lambda x_{1}+(1-\lambda) x_{2}\right) .
$$

If $T$ is single-valued, then the convexity of $T$ is exactly the same as the affine condition (see [10]). For $A \subseteq X$, we will denote

$$
T(A):=\{y \in Y: y \in T(x) \text { for some } x \in A\} .
$$

$T$ is said to be upper semicontinuous if for each $x \in X$ and each open set $V$ in $Y$ with $T(x) \subset V$, there exists an open neighborhood $U$ of $x$ such that $T(y) \subset V$ for some $y \in U$. Let $\mathcal{A}:=\left\{T \mid T: X \rightarrow 2^{X}\right\}$ be a family of multimaps on $X$ into $2^{X} . \mathcal{A}$ is a commutating family of multimaps if for each $x \in X$ and every pair $S, T$ in $\mathcal{A}$, we have $S(T(x))=T(S(x))$. Also, we denote the fixed point set $\mathcal{F}(T)$ as $\mathcal{F}(T):=\{x \in X: x \in T(x)\}$.

Theorem 2.1 ([11]) Let $K$ be a compact and star-shaped subset of a topological linear space $X$. Then every decreasing chain of nonempty, compact and star-shaped subsets of $K$ has a nonempty intersection that is compact and star-shaped.

Lemma 2.2 ([11]) Suppose that $K$ is a compact star-shaped subset of a topological linear space $X$ and $A$ is the corresponding star-core of $K$. Then $A$ is a compact convex subset of $A$.

Lemma 2.3 ([10]) Let X be a nonempty, compact and convex subset of a Hausdorfflocally convex space and let $T: X \longrightarrow 2^{X}$ be an upper semicontinuous multimap such that for each $x \in X, T(x)$ is nonempty, closed and convex. Then there exists a point $\bar{x} \in X$ such that $\bar{x} \in T(\bar{x})$.

Theorem 2.4 (Schauder [12]) Let X be a nonempty, compact and convex subset of a Hausdorff locally convex space $V$. If $T: X \rightarrow X$ is a continuous map, then $T$ has a fixed point. 


\section{Main results}

\subsection{Fixed point results}

Definition 3.1 Let $C$ be a convex subset of a locally convex space $X$. For $q \in C$ and $T$ : $C \longrightarrow 2^{C}, T$ is said to be $q$-convex if we have the following condition:

$$
T(\lambda q+(1-\lambda) x) \supseteq \lambda T(q)+(1-\lambda) T(x)
$$

for all $x \in C$ and $\lambda \in[0,1]$.

To establish the main results, we need the following lemma.

Lemma 3.2 Let $C$ be a star-shaped subset of a topological vector space $X$ and $T: C \longrightarrow 2^{C}$ be onto and $q$-convex with each $q$ in the star-core of $C$. If $S$ is the star-core of $C$, then $S$ is invariant under $T$.

Proof Suppose that $x_{o} \in S$. We will show that $T\left(x_{0}\right) \in S$. Let $t \in T\left(x_{0}\right), y \in C$ and $0 \leq \lambda \leq 1$. By hypothesis, there exists $x \in C$ such that $y \in T(x)$. Since $x_{0}$ is a star point of $C$, then $\lambda x_{0}+(1-\lambda) x \in C$. But $T$ is $q$-convex on $S$ and $C$ is invariant under $T$. Then we have

$$
\begin{aligned}
\lambda t+(1-\lambda) y & \in \lambda T\left(x_{0}\right)+(1-\lambda) T(x) \\
& \subseteq T\left(\lambda x_{0}+(1-\lambda) x\right) \\
& \in T(C) \subseteq C .
\end{aligned}
$$

Hence $S$ is invariant under $T$.

Now the main result of this section will be proved.

Theorem 3.3 Let $X$ be a nonempty compact and star-shaped subset of a locally convex Hausdorff space $V, C$ be the star-core of $X$ and $\mathcal{A}$ be a commutating family of upper semicontinuous multimaps such that for each $T \in \mathcal{A}, \mathcal{F}(T) \subseteq C$. Also, suppose that for each $x \in X, T(x)$ is a nonempty, closed and star-shaped subset of $X$ and $T$ is $p$-convex for each $p \in C$. Moreover, suppose that for each $x \in \mathcal{F}(T)$, we have the following condition:

$$
y \in S(x) \Rightarrow S(T(x)) \subseteq T(y), \quad \forall S, T \in \mathcal{A} .
$$

Then there is a point $\bar{x} \in X$ such that for each $T \in \mathcal{A}$, we have $\bar{x} \in \mathcal{F}(T)$.

Proof First, we try to confine the domain to a convex set. To do this, we apply Theorem 2.1, Lemma 3.2 and Zorn's lemma to obtain a set $M \subseteq X$ such that $M$ is minimal with respect to being nonempty, compact, star-shaped and invariant under each $S \in \mathcal{A}$. We show that for each $S \in \mathcal{A}, S(M)=M$. On the contrary, suppose $T \in \mathcal{A}$ is such that $T(M) \subset M$. Let $T(M)=N$. Since $T$ is p-convex, then it preserves the star-shapeness. Hence $N$ is starshaped. Also, $N$ is nonempty and compact. Suppose that $x \in N=T(M)$. Then there exist some $y \in M$ such that $x=T(y)$. Since $\mathcal{A}$ is commutative, for each $S \in \mathcal{A}$, we have $S(x)=$ $S(T(y))=T(S(y)) \in T(M)=N$. Hence, for each $S \in \mathcal{A}, S(N) \subseteq N$. So, $N \subset M$ is a nonempty, compact and star-shaped subset of $X$ that is invariant under each $S \in \mathcal{A}$. But this is a 
contradiction to the minimality of $M$, and so for each $S \in \mathcal{A}$, we have $S(M)=M$. Let $M$ be the star-core of $X$. In view of Lemma 2.2, $M$ is a nonempty, compact and convex subset of $X$ that is invariant under any $S \in \mathcal{A}$. So, we can replace $M$ by $X$ and hence we may suppose that $X$ is nonempty, compact and convex. For each $T \in \mathcal{A}$, by hypothesis and Lemma 2.3, $T$ is nonempty and closed in $X$. Suppose that $x_{1}, x_{2} \in \mathcal{F}(T)$ and $\lambda \in[0,1]$. By $p$-convexity of $T$, we have

$$
\lambda x_{1}+(1-\lambda) x_{2} \in \lambda T\left(x_{1}\right)+(1-\lambda) T\left(x_{2}\right) \subseteq T\left(\lambda x_{1}+(1-\lambda) x_{2}\right) .
$$

Hence $\mathcal{F}(T)$ is convex. We claim that for each $S, T \in \mathcal{A}, \mathcal{F}(S) \cap \mathcal{F}(T)$ is nonempty, closed and convex in $X$. Consider the multimap $S: \mathcal{F}(T) \longrightarrow 2^{X}$ which is the restriction of $S$ on $\mathcal{F}(T)$. We will show that for each $x \in \mathcal{F}(T), S(x) \subseteq \mathcal{F}(T)$. Suppose there exists a point $y \in S(x)$ such that $y \notin T(y)$. According to commutativity in $\mathcal{A}$ and condition (1), we have

$$
y \in S(x) \subseteq S(T(x))=T(S(x)) \subseteq T(y)
$$

and this is a contradiction. Then the map $S: \mathcal{F}(T) \longrightarrow 2^{\mathcal{F}(T)}$ is an upper semicontinuous multimap such that for each $x \in \mathcal{F}(T), F(x)$ is nonempty, closed and convex. Now by Lemma 2.3, there exists an element $\bar{x} \in \mathcal{F}(T)$ such that $\bar{x} \in S(\bar{x})$. Similarly, for each triple $\{S, T, W\} \subset \mathcal{A}$, by considering the multimap $W: \mathcal{F}(T) \cap \mathcal{F}(S) \longrightarrow 2^{X}$, we claim that for each $x \in \mathcal{F}(T) \cap \mathcal{F}(S), W(x) \subset \mathcal{F}(T) \cap \mathcal{F}(S)$. To prove the claim, suppose that $y \in W(x)$ such that $y \notin T(y)$ or $y \notin S(y)$. Let $y \notin S(y)$. By commutativity of $\mathcal{A}$ and by equation (1), we have

$$
y \in W(x) \subseteq y \in W(T(x))=T(W(x)) \subseteq T(y),
$$

which is a contradiction. So, the multimap $W: \mathcal{F}(T) \cap \mathcal{F}(S) \longrightarrow 2^{\mathcal{F}(T) \cap \mathcal{F}(S)}$ is upper semicontinuous, and for each $x, W(x)$ is nonempty, closed and convex. Therefore, there exists $\bar{x} \in \mathcal{F}(T) \cap \mathcal{F}(T)$ such that $\bar{x} \in W(\bar{x})$. By induction, we obtain a family $\{\mathcal{F}: T \in \mathcal{A}\}$ in $X$ which has the finite intersection property. We know that each $\mathcal{F}$ is nonempty and compact. So, there exists $\bar{x} \in X$ such that $\bar{x} \in \bigcap_{T \in \mathcal{A}} T$.

In the sequel, we see some important corollaries that can be implied as the results of the above theorem.

In Theorem 3.3, when $\mathcal{A}$ is a commutating family of single-valued maps, then the convexity assumption on $\mathcal{A}$ is actually the affine condition. Also, we can easily see that equation (1) automatically holds. So, we have the following results.

Corollary 3.4 (Markov-Kakutani) Let $X$ be a nonempty, compact and convex subset of a Hausdorfflocally convex space and let $\mathcal{A}$ be a commutating family of affine mappings. Then there exists an element $\bar{x} \in X$ such that $f(\bar{x})=\bar{x}$ for each $f \in \mathcal{A}$.

Corollary 3.5 ([11]) Let $K$ be a compact star-shaped subset of a locally convex space $V$. Suppose $F$ is a commutative family of continuous affine mappings of $K$ into itself. Then $F$ has a common fixed point in $K$.

If we apply the technique introduced in the first part of proof in Theorem 3.3, we have the following extension of Lemma 2.3. 
Corollary 3.6 Let X be a nonempty, compact and star-shaped subset of a Hausdorfflocally convex space $V$ and let $T: X \longrightarrow 2^{X}$ be an upper semicontinuous multimap such that for each $x \in X, T(x)$ is nonempty, closed and convex. Then there exists a point $\bar{x} \in X$ such that $\bar{x} \in T(\bar{x})$.

Also, if $T$ is a single-valued map, we have the following extension of the Schauder fixed point theorem.

Corollary 3.7 Let X be a nonempty, compact and star-shaped subset of a Hausdorfflocally convex space $V$. If $T: X \rightarrow X$ is a continuous map, then $T$ has a fixed point.

\subsection{Applications for the Cauchy problem}

Suppose that $(X, \tau)$ is a Hausdorff locally convex space and $(Y, d)$ is a complete metric space. Take a set $A \subseteq X$, a mapping $C: A \longrightarrow C(A) \subseteq Y$ and a mapping $T$ defined at least on the set $A \times C(A)$ and having the range in $X$. We are interested in the existence of a point $x \in A$ such that

$$
x=T(x, C(x)) .
$$

Definition 3.8 The family $\{T(\cdot, y): y \in Y\}$ is said to be equicontractive if there exists a $k \in[0,1)$ such that

$$
p_{\alpha}\left(T\left(x_{1}, y\right)-T\left(x_{2}, y\right)\right) \leq k p_{\alpha}\left(x_{1}-x_{2}\right)
$$

for each $p_{\alpha} \in A^{*}(\tau)$ and for all $\left(x_{1}, y\right),\left(x_{2}, y\right)$ in the domain of $T$.

Theorem 3.9 Suppose that A is a compactstar-shaped subset of a Hausdorfflocally convex space $X$ and $C: A \longrightarrow Y$ is a continuous operator. Let

$$
T: A \times C(A) \longrightarrow A
$$

be a continuous operator such that the family $\{T(\cdot, y): y \in C(A)\}$ is equicontractive. Then operator equation (2) admits a solution in A.

Proof Suppose $y \in C(A)$ is arbitrary. Since the operator

$$
x \rightarrow T(x, y): A \longrightarrow A
$$

is a contraction, there exists a unique point $x=F(y) \in A$ that satisfies the operator equation

$$
T(F(y), y)=F(y) .
$$

Now we will show that the mapping $y \rightarrow F(y): C(A) \rightarrow A$ is continuous. To do this, suppose that $\left(y_{\beta}\right)$ is a net in $C(A)$ such that $y_{\beta} \rightarrow y_{0}$. Then for each $p_{\alpha} \in A^{*}(\tau)$, we have

$$
\begin{aligned}
p_{\alpha}\left(F\left(y_{\beta}\right)-F\left(y_{0}\right)\right) & =p_{\alpha}\left(T\left(F\left(y_{\beta}\right), y_{\beta}\right)-T\left(F\left(y_{0}\right), y_{0}\right)\right) \\
& \leq p_{\alpha}\left(T\left(F\left(y_{\beta}\right), y_{\beta}\right)-T\left(F\left(y_{0}\right), y_{\beta}\right)\right)
\end{aligned}
$$




$$
\begin{aligned}
& +p_{\alpha}\left(T\left(F\left(y_{0}\right), y_{\beta}\right)-T\left(F\left(y_{0}\right), y_{0}\right)\right) \\
\leq & k p_{\alpha}\left(F\left(y_{\beta}\right)-F\left(y_{0}\right)\right)+p_{\alpha}\left(T\left(F\left(y_{0}\right), y_{\beta}\right)-T\left(F\left(y_{0}\right), y_{0}\right)\right) .
\end{aligned}
$$

Therefore, we have

$$
p_{\alpha}\left(F\left(y_{\beta}\right)-F\left(y_{0}\right)\right) \leq(1-k)^{-1} p_{\alpha}\left(T\left(F\left(y_{0}\right), y_{\beta}\right)-T\left(F\left(y_{0}\right), y_{0}\right)\right),
$$

and this proves the continuity of $F$. Now, the operator FoC is a self-mapping on $A$ that is continuous and affine. Hence, by Corollary 3.7 there exists a point $\bar{x} \in A$ such that $F(C(\bar{x}))=\bar{x}$. But this means that

$$
T(\bar{x}, C(\bar{x}))=T(F(C(\bar{x})), C(\bar{x}))=F(C(\bar{x}))=\bar{x} .
$$

Before continuing, we replace equation (3) by the following:

$$
T(x, C(x)) \in A, \quad x \in A
$$

In the sequel, an application of the above theorem will be presented, but first we remind some preliminaries.

Let $I$ be an interval of the real line of the form $[0, t)$, where $t$ is a positive number or $\infty$. Let $X$ be a Banach space $C_{b}(I, \mathbb{R})$ of all continuous and bounded functions $x: I \rightarrow \mathbb{R}$ endowed with a sup-norm $\|\cdot\|$. By $B(o, r)$, we will denote the ball in $X$ centered at zero and having radius $r(>0)$. Consider the following initial value problem (Cauchy problem):

$$
\left\{\begin{array}{l}
x^{\prime}(t)=g(t)+F(t) x(t)\left[\int_{0}^{t} D(t-s) x(s) d s\right]^{\eta}, \quad t \in I=[0, t) \\
x(0)=0
\end{array}\right.
$$

in the real scalar case, where $\eta>0$. We will show the existence of a solution $x \in X$ for equation (5). Suppose that $g, F, D \in L_{1}(I, \mathbb{R})$ and let $\|\cdot\|_{1}$ stand for the usual $L_{1}$-(semi)-norm. Also, assume that $\|F\|_{1}\|D\|_{1}>0$. For any fixed $K_{1} \in\left(0,\|F\|_{1}^{\frac{-1}{\eta}}\|D\|_{1}^{-1}\right)$, set $N=\|F\|_{1}^{\eta}\|D\|_{1}^{\eta}$ and $M_{1}=K_{1}\left(1-\|F\|_{1} N\right)$. By these assumptions, we have the following theorem.

Theorem 3.10 Let the above conditions hold. If $g \in L_{1}(I, \mathbb{R})$ and $\|g\|_{1} \leq M_{1}$, then equation (5) admits a (global) solution $x \in B\left(0, K_{1}\right)$.

Proof If we define the operator $C: X \rightarrow X$ by

$$
(C x)(t)=\left[\int_{0}^{t} D(t-s) x(s)\right]^{\eta}
$$

then $C$ is continuous. Suppose that $A=\left\{x \in X:\|x\| \leq K_{1}\right\}$. A simple calculation shows that $C$ maps $B\left(0, K_{1}\right)$ into the ball $B(0, N)$. Now we consider the operator $T: A \times B(0, N) \longrightarrow X$ as defined by

$$
T(x, y)(t)=\int_{0}^{t} g(s) d s+\int_{0}^{t} F(s) x(s) y(s) d s, \quad t \in I .
$$


We will show that $T$ has the condition of Theorem 3.9. For each $x \in A$, the operator $x \longrightarrow$ $T(x, C(x))$ is continuous and if $y=C(x)$, then for each $t \in I$, we have

$$
|T(x, y)(t)| \leq\|g\|_{1}+K_{1}\|F\|_{1} N \leq K_{1}\left(1-\|F\|_{1} N\right)+K_{1}\|F\|_{1} N=K_{1} .
$$

Hence for each $x \in A$, we have $T(x, C(x)) \in A$. Also, for each $x_{1}, x_{2} \in A, y \in C(A)$ and $t \in I$, we have

$$
\left|T\left(x_{1}, y\right)(t)-T\left(x_{2}, y\right)(t)\right| \leq \int_{0}^{t}|F(s)|\left|x_{1}(s)-x_{2}(s)\right||y(s)| d s \leq\left\|x_{1}-x_{2}\right\|
$$

where $k=\left\|F_{1}\right\|_{1} N<1$. This proves the equicontractivity condition and hence the proof is complete.

\section{Competing interests}

The authors declare that they have no competing interests.

\section{Authors' contributions}

All authors conceived of the study, participated in its design and coordination, drafted the manuscript, participated in the sequence alignment, and read and approved the final manuscript.

\section{Author details}

${ }^{1}$ Department of Mathematics, Faculty of Sciences, Yasouj University, Yasouj, 75914-353, Iran. ${ }^{2}$ Department of Mathematics, Research Institute for Natural Sciences, Hanyang University, Seoul, 133-791, South Korea.

Received: 2 September 2012 Accepted: 9 January 2013 Published: 22 February 2013

\section{References}

1. Aneke, SJ: Common fixed point of an arbitrary family of nonexpansive mappings in a locally convex topological vector spaces. Bull. Math. Anal. Appl. 2, 12-14 (2010)

2. Chen, YQ: Fixed points for convex continuous mappings in topological vector spaces. Proc. Am. Math. Soc. 129 2157-2162 (2000)

3. Hajji, A, Hanebaly, E: Commutating mappings and $\alpha$-compact type fixed point theorems in locally convex spaces. Int. J. Math. Anal. 1, 661-680 (2007)

4. Aubin, JP: Mathematical Methods of Game an Economic Theory. North-Holland, Amsterdam (1979)

5. Kim, WK, Yuan, GXZ: New existence of equilibria in generalized SSC. Comput. Math. Appl. 3, 751-758 (2002)

6. Neammanee, K, Kaewkhao, A: Fixed point theorems of multi-valued Zamfirescu mapping. J. Math. Res. 2, 150-156 (2010)

7. Kothe, G: Topological Vector Spaces-I. Springer, New York (1969)

8. Vijayaraju, P, Marudai, M: Some results on common fixed points and best approximations. Indian J. Math. 40, 233-244 (2004)

9. Nikodem, K: On midpoint convex set-valued functions. Aequ. Math. 33, 46-56 (1987)

10. Smart, DR: Fixed Point Theorems. Cambridge University Press, Cambridge (1974)

11. Hu, T, Heng, WS: An extension of Markov-Kakutani fixed point theorem. Indian J. Pure Appl. Math. 32, 899-902 (2001)

12. Schauder, J: Der fixpunktsatz in funktionalraumen. Stud. Math. 2, 171-180 (1930)

doi:10.1186/1687-1812-2013-37

Cite this article as: Goudarzi et al.: Common fixed point theorems for multimaps in locally convex spaces and some applications. Fixed Point Theory and Applications 2013 2013:37. 